\title{
Hermeneutics as the Methodology of Interpretation of Languages and Texts of Extraterrestrial Intelligence
}

\author{
Vira Dubinina \\ Ph.D., Associate Professor, Ukrainian Medical Stomatological Academy (Poltava, Ukraine) \\ E-mail: vera.dubinina777@gmail.com \\ https://orcid.org/0000-0001-8024-9823
}

Olga Tsybulco
Ph.D., Associate Professor, Mariupol State University (Mariupol, Ukraine)
E-mail: ostsybulco@ukr.net
https://orcid.org/0000-0003-1297-5465

In the paper, the authors emphasized the growth of number of publications in the authoritative scientific journals in which the fact of existence of extraterrestrial intelligence is approved and the features of communication with it are considered as well. All incoming information, which may relate to manifestations of extraterrestrial intelligence, a candidate SETI signal, is evaluated according to the Rio scale. The authors proposed to call a candidate SETI signal, which had reached according to the Rio scale maximum assessment - the SETI signal. The SETI signal is the established and scientifically proved fact of receiving a fragment of the text or the text of extraterrestrial intelligence. To interpret the SETI signal, the authors proposed to use the possibilities of hermeneutics. In the paper, the authors answered two questions: "IS it appropriate to use hermeneutics to interpret the SETI signal?" and "Does hermeneutics have any ability to interpret the SETI signal?" The authors showed briefly how to use the hypothetico-deductive method, or in what way it is possible to carry out interpretation of the SETI signal in five steps using the example. As a basis, the authors used the ideas of Chrysostomos Mantzavinos.

Keywords: hermeneutics; extraterrestrial intelligence; the Rio scale; SETI signal; hypotheticodeductive method; interpretation

Received: October 15, 2018; accepted: November 28, 2018

Philosophy and Cosmology, Volume 22, 2019: 101-108.

https://doi.org/10.29202/phil-cosm/22/8

(C) Dubinina, Vira, 2019

(C) Tsybulco, Olga, 2019 


\section{Introduction}

For the last two decades, more and more publications have appeared in the authoritative scientific journals in which the fact of existence of extraterrestrial intelligence is approved and the features of communication with it are considered as well. For example, in the article Calculating the Probability of Detecting Radio Signals from Alien Civilizations the author uses the Drake equation as the obvious starting point, and logically identifying and enumerating constraints of interstellar radio communications for justification of probability of detecting a genuine alien radio signal [Horvat, 2006]. In the article The Breakthrough Listen Search for Intelligent Life a team of astronomers presents the target selection for the Breakthrough Listen search for extraterrestrial intelligence during the first year of observations at the Green Bank Telescope, Parkes Telescope, and Automated Planet Finder [Isaacson et al., 2017].

In the paper Rio 2.0: Revising the Rio Scale for SETI Detections the Rio scale and options of its improvement are considered. The Rio scale assigns scores to signals detected in searches for extraterrestrial intelligence (SETI), which characterizes both the consequences of a signal and the probability the signal is truly from ETI, in an easily digestible format for laypeople to interpret. The authors of the article note, "In the 17 years since its construction, the number of groups actively conducting searches for evidence of intelligent life beyond the Earth has increased significantly, and theoretical work has established a new suite of observables that are capable of revealing the presence of ETI in a range of astronomical observations" [Forgan et al., 2018]. In this paper, authors present (and encourage the SETI community to adopt) a single set of consistent terminology for discussing signals [Forgan et al., 2018].

In the paper Psycholinguistics and the Search for Extraterrestrial Intelligence the author combines the current achievements of psycholinguistics and neurophilosophy and answers the question: how psycholinguistics is able to help us in our search for Extraterrestrial Intelligence (SETI). The author draws attention to, first of all, the relationship between psycholinguistics and neuroscience, cognitive science, linguistics, and studies it with a focus on neurolinguistics [Krotenko, 2017].

As we see, in modern science the search for Extraterrestrial Intelligence (SETI) is carried out in two strategic directions:

1. The search and assignment of scores according to the Rio scale (giving of assessment on the Rio scale) signals detected in searches for extraterrestrial intelligence (SETI);

2. Consideration of opportunities of communication and interpretation of languages and texts of Alien Civilizations.

In this paper, we will consider the possibilities of using hermeneutics as the methodology of interpretation of languages and texts.

\section{Quantitative and qualitative assessment of a candidate SETI signal}

In the $21^{\text {st }}$ century, the search for Extraterrestrial Intelligence has reached a new level of development. It ceased to be considered as pseudo-scientific knowledge. Now the research teams on the Earth and in space study it. Searches for extraterrestrial intelligence are conducted not only by the telescopes on the Earth but space telescopes, which are derived in the Earth orbit and in solar orbit. Currently, more than a dozen space telescopes collect data in the frequency ranges: gamma ray, $\mathrm{x}$-ray, ultraviolet, visible, infrared, microwave and radio. In the book Space Telescopes: Capturing the Rays of the Electromagnetic, Neil English shows 
how different parts of the electromagnetic spectrum provide different information about the universe - and why specialized space observatories are needed. He discusses the future of space telescope technologies and the status of space telescopes that are now being built or on the drawing board [English, 2017].

All information, which can relate to manifestations of extraterrestrial intelligence, is estimated on the Rio scale. The idea of assessment of a candidate SETI signal was first proposed in Rio de Janeiro, Brazil, in October 2000 by Ivan Almar and Jill Tarter. It reminds very much the Richter Scale for quantifying earthquake severity. The Rio Scale is an ordinal scale between zero and ten, used to quantify the impact of any public announcement regarding evidence of extraterrestrial intelligence. Specially, the website (http://www. setileague.org/iaaseti/rioscale.htm) has been created where one cannot only be acquainted with the criteria of the Rio scale, but also leave some messages according to this scale. The Rio Scale is constantly being improved, and the incoming a candidate SETI signal is analyzed and systematized. Rio Scale Calculator (http://www.setileague.org/iaaseti/riocalc. $\mathrm{htm}$ ) has been created, which allows to evaluate a candidate SETI signal from 0 (None) up to 10 (Extraordinary). In addition to the Rio scale in the search for Extraterrestrial Intelligence (SETI), two more scales are used:

1. Torino Scale. The Torino Scale, adopted by the International Astronomical Union in 1999. The two-dimensional Torino Scale takes into account both the potential damage from such an asteroid impact, and the probability that it will collide with Earth (https:// cneos.jpl.nasa.gov/sentry/torino_scale.html).

2. The San Marino Scale. This metric system was created in 2005 for quantifying the potential hazard associated with an active SETI experiment, or other aimed transmission from Earth into space (http://www.setileague.org/iaaseti/smiscale.htm).

Anyway, all three scales are designed for quantitative and qualitative assessment of a candidate SETI signal.

In 1960, International Academy of Astronautics (IAA) drafted the "Declaration of Principles Concerning Activities Following the Detection of Extraterrestrial Intelligence." The document has been endorsed by six international professional space societies and also constitutes an informal agreement among most of those carrying out SETI [Billingham et al., 1960]. The Earth civilization is not only looking for extraterrestrial civilizations, but also develops a policy of communication with them.

\section{The SETI signal and hermeneutics}

Further, we suggest to call a candidate SETI signal, which reached according to the Rio scale of the maximum assessment - the SETI signal. As we understand, the SETI signal is the established and scientifically proved fact of receiving a fragment of the text or the text of extraterrestrial intelligence. Probability of the SETI signal is rather high. It is indicated by the theory "Evolving Matter" proposed by Oleg Bazaluk [Bazaluk, 2016] and the philosophy of the cosmos as the new universal philosophical teaching about Being [Bazaluk \& Kharchenko, 2018].

Lidija Krotenko proved that the evolution of language is, in essence, a particular case of neuroevolution. If neuroevolution is considered through the theory "Evolving Matter", then the SETI signal has already something what is inherent in our earthly civilization. Neuroevolution, in particular the evolution of language, unites the earthly civilization with the extraterrestrial intelligence [Krotenko, 2017]. The problem of origin of the SETI signal does not come to the forefront any more. A priori, the fact of perception a candidate SETI signal 
as the SETI signal indicates one whole ontologic basis of a terrestrial and extraterrestrial civilization, neuroevolution, or noogenesis [Bazaluk, 2016]. The problem of interpretation of the SETI signal comes to the forefront. Interpretation of languages and texts, as we know, is a problem field of hermeneutics. Thus, we have to answer two questions:

1. Is it appropriate to use hermeneutics to interpret the SETI signal?

2. Does hermeneutics have any ability to interpret the SETI signal?

The review article about history and opportunities of hermeneutics is presented in the Stanford Encyclopedia of Philosophy [Mantzavinos, 2016]. Chrysostomos Mantzavinos notes, "As a methodological discipline, hermeneutics offers a toolbox for efficiently treating problems of the interpretation of human actions, texts and other meaningful material" [Mantzavinos, 2016]. Indeed, in practice, hermeneutics has proven its effectiveness. In essence, it restored and continues to restore for contemporaries a cultural context and the lost meanings of those times in which written language only arose. By fragments of texts, for example, we can recover the feature transformations of the basic meanings of Greek Paideia in the educational theories in the Middle Ages [Bazaluk, 2018]. Hermeneutic methods allow, for example, "to reconsider the soviet philosophical heritage for better understanding the real value of any philosophical conclusions and worldview-concerning statements which were made in the times of soviet ideology hegemony" [Tytarenko \& Rudenko, 2018]. On the scale of a terrestrial civilization, hermeneutics methods are irreplaceable. Chrysostomos Mantzavinos admits that a few decades ago indeed "Philosophical hermeneutics in the tradition of Heidegger and Gadamer and deconstructionism in the tradition of Derrida have challenged the possibility of achieving any kind of hermeneutic objectivity" [Mantzavinos, 2014]. However, this "postmodernist" discourse remained in history. Hermeneutics and its methods are no longer torn into two opposite processes: 1) the conceptualization process as a scientific process of restoration of incompatible meanings that can be focused on the idea of truth; 2) process of a deconstruction, i.e. dissolution of interpretation of any text in many equivalent constructions deserving attention allegedly.

Currently, hermeneutics is one and integral. It uses as the standards that act in the natural sciences as philosophical methods that also proved their effectiveness in the history of culture. Hermeneutics possesses all necessary tools for restoration of undercover meanings and a context of the written texts.

Two levels of analysis are distinguished in hermeneutics, the ontological and the epistemological. The difference between the two levels is in the sets of methods that are preferred for interpreting texts. In fact, the age-old "Verstehen vs. Erklären" debate is largely about this question: whether there is a distinct method for the apprehension of meaningful material, employable in the social sciences and the humanities (Geisteswissenschaften; Kulturwissenschaften), which deal with such material, i.e., Verstehen (understanding), or whether the general method employed in the natural sciences is successfully employable in the social sciences and humanities as well, i.e., Erklären (explanation) [Mantzavinos, 2016]. In any case, methodological monists and methodological dualists attract the most modern and most effective methods of interpretation of texts in hermeneutics. In fact, they turn hermeneutics into the applied science, which allows us to consider in texts, regardless of the time of their writing, much more meanings than it is presented in the composition of words and sentences. In the article Symptoms of Expertise: Knowledge, Understanding and Other Cognitive Goods the author has considered two main points: (I) The first point is methodological: Instead of attempting to give a classical analysis or reductive definition of the term "expertise", we should attempt an explication and look for what may be called symptoms of expertise. (II) 
The second point is substantial: Scholz recommends understanding as an important symptom of expertise [Scholz, 2018]. Scholz revealed opportunities of the hypothetico-deductive method that united intersubjective intelligibility, testability with the use of evidence, rational argumentation and objectivity. Scholz proved that modern hermeneutics concentrates in itself the highest standards of scientific and philosophical interpretation of the text and related events.

However, are these standards enough to interpret the SETI signal? Indeed, in the definition of hermeneutics by Chrysostomos Mantzavinos its scope is limited by "human actions and the products of such actions, most importantly texts" [Mantzavinos, 2016].

We are convinced that only the methods of hermeneutics, especially the hypotheticodeductive method, can qualitatively interpret the SETI signal. Mantzavinos set standards for text interpretation himself. These standards involve intersubjective intelligibility, testability with the use of evidence, rational argumentation, and making methodological decisions aiming at the attainment of truth, accuracy, simplicity and other epistemic values [Mantzavinos, 2014]. Let us consider how these standards will help interpret the SETI signal.

\section{Use of the hypothetico-deductive method for interpretation of the SETI signal}

Let us review an example of interpretation of the SETI signal by means of the hypotheticodeductive method. For example, one of space telescopes recorded a candidate SETI signal. According to the Rio scale, this signal was rated at a maximum of 10 points (Extraordinary). Accordingly, we have talked about the SETI signal and the necessity of its interpretation. We will carry out interpretation of the established SETI signal, using the sequence of actions, which were developed by Chrysostomos Mantzavinos [Mantzavinos, 2014]. The hypotheticodeductive method proposed by Mantzavinos focuses on the most effective scientific and philosophical methods of interpretation of the SETI signal.

The first step. Any SETI signal, as well as the author's text, has the definite meaning. This may be a fragment of a conversation intercepted by space telescopes, or a specific signal addressed to the Earth civilization, or something else. A first step is to identify this meaning by correctly reconstructing the fundamental elements of nexus which were laid by extraterrestrial intelligence. Mantzavinos suggests to establish at once the interpretive hypotheses. The interpretive hypotheses by themselves are conditional, hypothetical because it is not clear whether they can fulfil their basic epistemic aim, i.e., the identification of the meaning of the text. For interpretation of the SETI signal as the basic interpretive hypothesis, we use the theory "Evolving Matter" proposed by Oleg Bazaluk [Bazaluk, 2016]. In essence, Bazaluk suggests to consider neuroevolution as the universal phenomenon i.e. as a basis of any extraterrestrial civilization. If the universality of evolution is accepted as the interpretive hypothesis, then in order to interpret the SETI signal the methods of analytic philosophy of language: the "principle of charity", the "principle of humanity" become available, etc. The logical status of the listed principles and methods extends the amount of the interpretive hypothesis, due to which the quality of interpretation of the SETI signal increases significantly. It becomes possible to adapt the SETI signal to the history of culture of the Earth civilization, and to consider its interpretation in the meanings and terms of modern science and philosophy. The interpretive hypotheses involve in the interpretation of the SETI signal the existing wealth of hermeneutic methods and experience.

The second step. The interpretive hypotheses allow access to use in the interpretation of the SETI signal other statements consequences that could be (more easily) testable. For example, 
if one of the interpretive hypotheses is that the SETI signal is an extraterrestrial intelligence conversation fragment that was accidentally intercepted, then it is checked by other statements consequences, for example, if it is a conversation fragment, then why only a fragment, not the whole conversation, what is the probability of finding extraterrestrial intelligence at the point of interception of the conversation fragment, etc. As Mantzavinos notes, "Such observable consequences can normally be formulated when the interpretative hypothesis is embedded in a larger theoretical context, i.e. when one or more auxiliary hypotheses are employed" [Mantzavinos, 2014]. In the case of interpretation, these auxiliary hypotheses will greatly expand the understanding of the SETI signal and involve its consideration in interdisciplinary scientific discourse. If to return and review specifically our example of interpretation of the SETI signal, then the interpretative hypothesis is a signal of communication. In such a case, auxiliary hypotheses are: assessment of the technical development of extraterrestrial intelligence; the consequences of communication; the need for communication; etc.

The third step. After the interpretive hypotheses are formulated, and observable consequences are drawn from them with the use of deduction, they must be empirically tested. This is the meaning of the third step of the application of the hypothetico-deductive method. Check (test) of the interpretive hypotheses is carried out with use of available knowledge and methods. During testing attention is paid to the details, connections, features. The auxiliary hypotheses are tested, which testing is more accessible and reliable. If the SETI signal is interpreted as a communication signal, then auxiliary hypotheses confirming the interpretive hypotheses are checked. As Mantzavinos writes, "In any case it is important to emphasize that in listing the various features that are supposed to support the respective interpretive hypothesis, it is not only the number of features that is important; so are the type of features that we are dealing with" [Mantzavinos, 2014]. What are evidences there to argue that the SETI signal is a signal for communication? How reliable is this interpretation of the SETI signal from the point of view of the Earth civilization? What motives can lie behind the offer on communication? The variety of auxiliary hypotheses and the need of their proof the help of research techniques from the social sciences and humanities allow to achieve the main goal. The hypothetico-deductive method involves in interpretation of the SETI signal the vast experience of use of hermenettics as the methodology of interpretation of languages and texts.

The fourth step. When testing the interpretive hypotheses, the question arises how to separate good interpretations from bad ones. Mantzavinos offers, "The various reconstructions of nexuses of meaning offered are to be compared to each other with regard to their truth, and those to be preferred that most accurately reconstruct the respective nexuses of meaning" [Mantzavinos, 2014]. In essence, it is a question of evaluating interpretive hypotheses in relation to ideals absolutized in society. When the same set of interpretations estimates different ideals, its discrepancy will be found. Following Mantzavinos' logic, comparing every hypothesis of interpretation of the SETI signal with the absolutized ideals, we have an opportunity to select good interpretations. If we go back to our example and view the SETI signal as the intention of extraterrestrial intelligence to establish communication with the Earth civilization, then we should check the evidence sets of interpretive hypotheses and auxiliary hypotheses by comparing them with the absolute truths. For example, to carry out their comparative evaluation with the "idea of good" that dominates in the history of culture of the Earth civilization, proposed by Plato [Bazaluk, 2018], or with the esthetic values [Mantzavinos, 2014]. Just as a result of this assessment, "bad" interpretations will be set aside, and the interpretations that most accurately reconstruct the respective nexuses 
of meaning will remain. In particular, the interpretation of the SETI signal as the desire to establish partnerships between two civilizations, for a joint, mutually beneficial development of resources of the Universe.

The fifth step. The final step of the hypothetico-deductive method consists in "a multidimensional evaluation of the same hypothesis with respect to different values or of a set of hypothesis with respect to one value" [Mantzavinos, 2014]. From the offered interpretative hypotheses, only one is accepted that has more significant argumentation and corresponds most fully to the absolutized truths. If the SETI signal is interpreted as the offer of partnership from an extraterrestrial civilization, then this interpretation is not accepted as the approved truth, but it is considered as the possibility of criticism. Only the interpretation of the SETI signal open for criticism is capable to convey true meaning of the SETI signal. The openness of interpretation for criticism is the possibility of correction of mistakes and improvement of actions arising from the interpretation. Therefore, the last step of the hypothetico-deductive method is precisely the actual exposure of the chosen interpretation of the SETI signal for the cross-disciplinary analysis and comprehensive criticism.

Thus, using the example of the SETI signal, we considered the possibilities of the hypothetico-deductive method. We have proved that hermenetics can be considered as the methodology of interpretation the SETI signal.

\section{Conclusions}

As a result of our research, we came to the following conclusions:

1. We have stated the growth of number of publications in the authoritative scientific journals in which the fact of existence of extraterrestrial intelligence is approved and the features of communication with it are considered

2. We have recorded that at present, all information that may relate to manifestations of extraterrestrial intelligence is estimated by the Rio scale from 0 (None) to 10 (Extraordinary). The higher the value of a candidate SETI signal, the more fixed attention is paid to the detected signal from the scientific community.

3. We have proposed to call a candidate SETI signal, which reached on the Rio scale of the maximum assessment - the SETI signal. We have given the following definition of the SETI signal - it is an established and scientifically proved fact of receiving a fragment of the text or the text of extraterrestrial intelligence.

4. We believe that the Rio Scale, as well as the Torino Scale, and the San Marino Scale are not enough to interpret the SETI signal. The authors proposed to interpret the SETI signal with the hypothetico-deductive method. In the paper, the authors answered two questions: "Is it appropriate to use hermeneutics to interpret the SETI signal?" and "Does hermeneutics have any ability to interpret the SETI signal?"

At the end of the paper, we showed briefly how to use the hypothetico-deductive method on practice. We have shown in what way it is possible to interpret the SETI signal in five steps using scientific and philosophical methods of interpreting texts. We used the ideas of Chrysostomos Mantzavinos having extended their effect to the interpretation of the SETI signal.

\section{References}

Bazaluk, Oleg. The Theory of Evolution: From a Space Vacuum to Neural Ensembles and Moving Forward. Cambridge Scholars Publishing, 2016. 
Bazaluk, Oleg. The Feature Transformations of the Basic Meanings of Greek Paideia in the Educational Theories in the Middle Ages. Schole, Vol. 12.1, 2018: 243-258. https:// doi.org/10.21267/AQUILO.2018.12.10428

Bazaluk, Oleg, and Larysa Kharchenko. The Philosophy of the Cosmos as the New Universal Philosophical Teaching about Being. Philosophy and Cosmology, Volume 21, 2018: 6-13. https://doi.org/10.29202/phil-cosm/21/1

Billingham, John, Roger Heyns, David Milne, and Seth Shostak. Social Implications of the Detection of Extraterrestrial Civilization: Report on Workshops As the Cultural Aspects of Seti. SETI Press, 1999.

English, Neil. Space Telescopes: Capturing the Rays of the Electromagnetic. Springer, 2017. Forgan, Duncan, Jason Wright, Jill Tarter, Eric Korpela, Andrew Siemion, Iván Almár, and Elisabeth Piotelat. Rio 2.0: Revising the Rio Scale for SETI Detections. International Journal of Astrobiology, 2018, pp. 1-9. https://doi.org/10.1017/S1473550418000162

Horvat, Marko. Calculating the Probability of Detecting Radio Signals from Alien Civilizations. International Journal of Astrobiology, Volume 5, Issue 2 April 2006: 143-149. https://doi.org/10.1017/S1473550406003004

Isaacson, Howard, Andrew P. V. Siemion, Geoffrey W. Marcy, Matt Lebofsky, Danny C. Price, David MacMahon, Steve Croft, David DeBoer, Jack Hickish, Dan Werthimer, Sofia Sheikh, Greg Hellbourg, and J. Emilio Enriquez. The Breakthrough Listen Search for Intelligent Life: Target Selection of Nearby Stars and Galaxies. Astronomical Society of the Pacific, Volume 129, Number 975, 2017, pp. 054501.

Krotenko, Lidija. Psycholinguistics and the Search for Extraterrestrial Intelligence. Philosophy and Cosmology. Volume 19, 2017: 110-116.

Mantzavinos, Chrysostomos. Text Interpretation as a Scientific Activity. Journal for General Philosophy of Science, 45, 2014: 45-58.

Mantzavinos, Chrysostomos. Hermeneutics. The Stanford Encyclopedia of Philosophy, 2016. https://plato.stanford.edu/entries/hermeneutics/

Tytarenko, Vadym and Sergii Rudenko. Kant's Studies in Ukrainian Philosophy of Soviet Period. Future Human Image, Volume 9, 2018: 107-114. https://doi.org/10.29202/ fhi/9/11

Scholz, Oliver R. Symptoms of Expertise: Knowledge, Understanding and Other Cognitive Goods. Topoi. Volume 37, Issue 1, 2018: 29-37. https://doi.org/10.1007/s11245-0169429-5 